\title{
La provincia en Boyacá: unidad territorial, histórico-funcional de planificación en la gestión del desarrollo regional endógeno, 2004-2011*
}

\author{
The province in Boyacá: \\ territorial, historical and functional unit of \\ planning in the management of endogenous \\ regional development, 2004-2011
}

\footnotetext{
Politóloga e internacionalista, candidata a magíster en Educación de la Universidad de los Andes. Docente de la Universidad del Rosario. Bogotá, Colombia. Correo electrónico: estupinan.lorena@urosario.edu.co.
} 


\section{Resumen}

Este artículo analiza la provincia como dimensión territorial del desarrollo en Boyacá. En primer lugar, se describe la provincia como unidad territorial (lugar concreto de acción), histórica (trascendente), funcional (con usos específicos); en segundo lugar, se expone el concepto de planificación en la gestión del desarrollo regional endógeno para, finalmente, analizarlo desde lo provincial. Así, se establece que para "alcanzar un cambio social sostenido que lleve al perfeccionamiento de una región", es necesario partir de la activación de las capacidades internas, lo que incluye la organización del territorio, que en Boyacá es la provincia, y que es desde ella, que se debe planificar el desarrollo departamental.

Palabras clave: provincia, desarrollo endógeno, funcional, histórico, planificación.

JEL: O18, O21, Q58, R58

\section{Abstract}

This article analyzes the province as a territorial dimension of development in Boyacá. First, the province is described as a territorial (specific site of action), historical (transcendent) and functional unit (with specific uses). Secondly, the concept of planning in the management of endogenous regional development is exposed, in order to analyze it from the province. Therefore, it is established that "to achieve a sustainable social change that will lead in the development of a region" is necessary to start from the activation of the internal capabilities including the organization of the territory, which in Boyacá is the province and that is from the province that the departmental development should be planned.

Keywords: province, endogenous development, functional, historical, planning. 


\section{INTRODUCCIÓN}

El reconocimiento de lo complejo

e integral del desarrollo implica

la identificación de las relaciones

directas e indirectas que existen entre lo político, lo social, lo económico, lo cultural, lo ambiental y todo lo relativo al nivel de la conciencia humana.

Asimismo, significa comprender las interrelaciones de dichos ámbitos en el marco del desarrollo colectivo, local y regional, para que desde una mirada interdisciplinaria se pueda responder a las necesidades reales de las comunidades (Universidad del Rosario, 2009)

El presente artículo tiene como objetivo identificar, a través del análisis de los planes de desarrollo 2004-2007 y 20082011, la provincia en Boyacá como unidad territorial, histórico-funcional (Figura 1) de la planificación en la gestión del desarrollo regional endógeno.
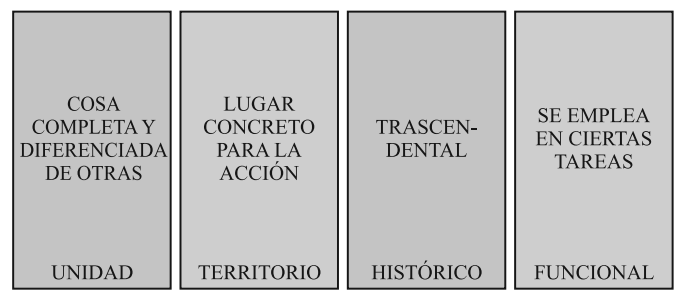

LA PROVINCIA EN BOYACÁ COMO

UNIDAD TERRITORIAL HISTÓRICO-FUNCIONAL

DE PLANIFICACIÓN EN LA GESTIÓN DEL DESARROLLO REGIONAL ENDÓGENO. 2004-2010

Figura 1. Red categorial.

Fuente: elaboración de la autora. 
Este objetivo, de tipo descriptivoanalítico-interpretativo, se desarrolla a través de tres apartados.

En primer lugar, se describe lo provincial a partir de las características naturales del terreno y desde la presencia de diferentes comunidades humanas que lo han habitado a lo largo de la historia; asimismo, se describe el uso otorgado en los ámbitos político y administrativo en el departamento. En segundo lugar, se expone el concepto de planificación en la gestión del desarrollo regional endógeno. Finalmente, se analiza e interpreta el rol de la provincia como unidad territorial, histórico-funcional de la planificación en la gestión del desarrollo regional endógeno en los más recientes planes de desarrollo departamentales.

De esta manera, se verifica la relación entre las dos variables que van a estudiarse. Por un lado, la provincia en Boyacá y por el otro, la planificación en la gestión del desarrollo regional endógeno, haciendo énfasis en la organización de los territorios como agente del desarrollo.
Se espera que el lector aprecie este escrito como una contribución a la diversidad que caracteriza los estudios sobre el desarrollo y, de paso, reconozca el compromiso de la autora por tratar de generar un efecto en su comunidad, en la realidad de la que es parte a través del aporte a su comprensión y de ser posible a su mejoramiento; lo anterior, como un propósito básico de la investigación en ciencias sociales.

\section{LA PROVINCIA EN BOYACÁ}

La provincia será entendida como una unidad territorial ${ }^{1}$ (lugar concreto para la acción) de distribución estratégica para la gestión (que cumple unas funciones ${ }^{2}$ ) conformada por municipios de características homogéneas con fundamento histórico (que trasciende) $y$ que de manera natural o institucional han definido una cabecera dispuesta como el principal centro de servicios (Gobernación de Boyacá, 2008).

Dicha provincia tiene como propósito profundizar la identidad de la población, coordinar actividades de los municipios asociados e impulsar proyectos de

\footnotetext{
Se apela a la noción de unidad territorial para diferenciar la Provincia de las entidades territoriales que sí lo son según la ley. Según el artículo 286 de la Constitución Política "son entidades territoriales, los departamentos, los distritos, los municipios y los territorios indígenas..." (Ministerio de Justicia y del Derecho, 1998, p. 147).

2 En lo que se refiere a las provincias y sus características, el artículo 321 de la Constitución, dice que "las provincias se constituyen con municipios o territorios indígenas circunvecinos, pertenecientes a un mismo departamento" (Ministerio de Justicia, 1998, p. 166). Se indica además que "la ley dictará el estatuto básico y fijará el régimen administrativo de las provincias que podrían organizarse para el cumplimiento de las funciones que les deleguen entidades nacionales o departamentales y que les asignen la ley y los municipios que las integran" (1998, p. 166). Y también indica que "las provincias serán creadas por ordenanza a iniciativa del gobernador, de los alcaldes de los respectivos municipios o del número de ciudadanos que determine la ley y que para el ingreso a una provincia ya constituida deberá realizarse una consulta popular en los municipios interesados" (1998, p. 166).

3 "La provincia únicamente por disposición legal puede obtener el carácter de nivel territorial" (Ministerio de Justicia y del Derecho, 1998. p. 147)
} 
interés departamental ${ }^{3}$. En el caso del departamento de Boyacá, la provincia es y ha sido un elemento fundamental de la "psicología profunda del boyacense" (Ocampo, 1997, p.60) y por lo tanto, en su modo de organizarse y relacionarse con el medio ambiente, es necesario ver al boyacense como parte de una provincia.

Según Javier Ocampo, las doce provincias que integran el departamento de Boyacá, son el resultado de la subdivisión de las cuatro regiones naturales que surgen en el territorio boyacense y que "conservan entre sí cierta trabazón ideal urdida por el origen racial y las costumbres todavía coloniales" (1997, p.26-27). Asimismo, pero desde el punto de vista de su origen institucional, la provincia fue la adaptación de la organización políticoadministrativa española en los territorios de ultramar, lo que llevó a la formación de "nuevas unidades étnico-políticas y al establecimiento de una nueva sociedad histórico-cultural de carácter mestizo" (1997, p. 56).

\section{La provincia como contenedor: un hecho natural}

El modo en que el territorio boyacense se ha configurado es por supuesto el resultado de unas condiciones naturales específicas.

El relieve del territorio de Boyacá pertenece al sistema andino, distinguiéndose a nivel macro cuatro unidades morfológicas: primero, el valle del río Magdalena o región de occidente; segundo, la cordillera Oriental o región del norte; tercero, el altiplano o región central, y cuarto, el piedemonte de los Llanos Orientales o región de oriente.

La Región Central (sic), llamada también altiplanicie Cundiboyacense (sic) ocupa el $70 \%$ del territorio boyacense. Se caracteriza porque en ella se concentra la mayor parte de la población, las ciudades y aldeas del Departamento (sic). Sus climas oscilan entre templado, frío y paramuno. Es zona minifundista y en donde se localiza el poder político y el área industrial. En esta región se concentró el mayor porcentaje de la población indígena chibcha y se localizaron los grupos españoles en la época colonial hispánica. Es el eje geopolítico y socio económico de Boyacá.

La Región de Oriente (sic), corresponde al Valle de Tenza, Lengupá y el piedemonte llanero de la vertiente oriental de la Cordillera (sic), se extiende hacia los Llanos de Casanare. El mayor concentramiento (sic) de la población se encuentra en el Valle de Tenza; el resto de la región... posee baja densidad demográfica.

La Región de Occidente (sic),

"La provincia únicamente por disposición legal puede obtener el carácter de nivel territorial" (Ministerio de Justicia y del Derecho, 1998. p. 147). 
corresponde a las tierras de la vertiente Occidental (sic) de la Cordillera (sic) Oriental, hacia el Río Magdalena. Ocupa el 15\% de la superficie de Boyacá. En esta región se encuentra el Territorio Vásquez, con densidad relativamente baja.

La región del Norte (sic), se extiende en las tierras del cordón magistral de la Cordillera (sic) Oriental, la vertiente del río Chicamocha y las tierras de la Tunebia en donde se encuentran los últimos sobrevivientes indígenas en Boyacá. (Gobernación de Boyacá, 2008, p. 8-11)

A su turno, estas cuatro unidades se dividen en diez subregiones naturales, dentro de las que se han establecido las doce provincias integradas por municipios con vínculos históricos, sociales y territoriales particulares, como se sigue:

La altiplanicie central, ahí encontramos las provincias de:

- Centro, integrada por los municipios de Tunja, Chivatá, Chíquiza, Cómbita, Cucaita, Motavita, Oicatá, Samacá, Siachoque, Sora, Soracá, Sotaquirá, Toca, Tuta y Ventaquemada.

- Tundama, integrada por los municipios de Belén, Busbanzá, Cerinza, Corrales, Duitama, Floresta, Paipa, Santa Rosa de Viterbo y Tutasá.

- Sugamuxi, integrada por los municipios de Aquitania, Cuítiva, Firavitoba,
Gámeza, Iza, Labranzagrande, Mongua, Monguí, Nobsa, Pajarito, Pesca, Tibasosa, Tópaga, Tota y Sogamoso.

El altiplano de Ramiriquí, allí se encuentra la provincia de:

- Márquez, integrada por los municipios de Boyacá, Ciénaga, Jenesano, Nuevo Colón, Ramiriquí, Rondón, Tibaná, Turmequé, Úmbita y Viracachá.

La subregión de Paz de Río, Tasco y Socha, aquí se ubica la provincia de:

- Valderrama, integrada por los municipios de Betéitiva, Chita, Jericó, Paz del Río, Paya, Pisba, Socha, Socotá y Tasco.

La subregión del norte y Gutiérrez, que da lugar a las provincias de:

- Norte, integrada por los municipios de Boavita, Covarachía, La Uvita, San Mateo, Sativanorte, Sativasur, Soatá, Susacón y Tipacoque.

- Gutiérrez, integrada por los municipios de Chiscas, Cubará, El Cocuy, Guacamayas, Güicán, El Espino y Panqueba.

El Valle del Magdalena o Territorio Vásquez que junto a la subregión denominada la Vertiente Occidental Andina y la subregión del Valle de Chiquinquirá, son lugar de emplazamiento de la provincia de: 
- Occidente, integrada por los municipios de Briceño, Buenavista, Caldas, Chiquinquirá, Coper, La Victoria, Maripí, Muzo, Otanche, Pauna, Puerto Boyacá, Quípama, Saboyá, San Miguel de Sema, San Pablo de Borbur y Tununguá.

La subregión de Villa de Leyva y Moniquirá, lugar de la provincia de

- Ricaurte, integrada por los municipios de Arcabuco, Chitaraque, Gachantivá, Moniquirá, Ráquira, Sáchica, Santana, San José de Pare, Santa Sofía, Sutamarchán, Tinjacá, Togüí y Villa de Leyva.
La subregión del Valle de Tenza en donde se ubica las provincias de:

- Neira, integrada por los municipios de Chinavita, Garagoa, Macanal, San Luis de Gaceno y Santa María.

- Oriente, integrada por los municipios de Almeida, Chivor, Guateque, Guayatá, La Capilla, Pachavita, Somondoco, Sutatenza y Tenza.

Y la subregión de Lengupá donde se ubica la provincia de:

- Lengupá, integrada por los municipios de Berbeo, Campohermoso, Miraflores, Páez, San Eduardo y Zetaquira. (Ocampo, 1997, p. 29-35)

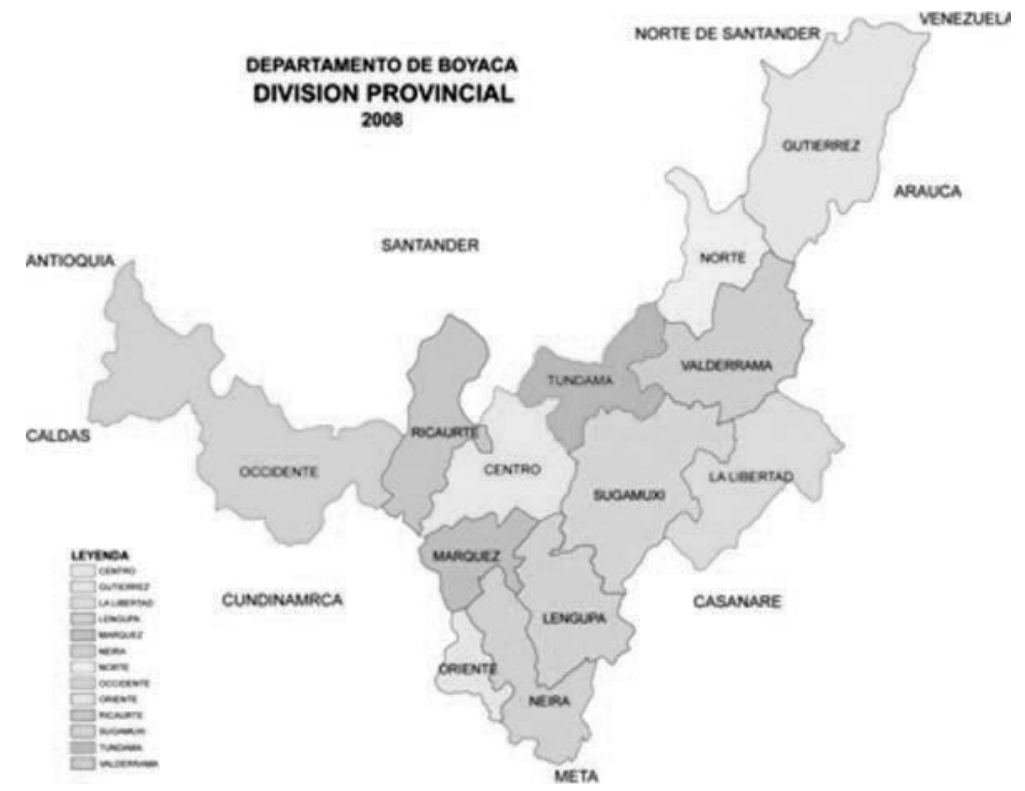

Figura 2. Mapa de Boyacá por provincias. Fuente: Gobernación de Boyacá (2004). 
Respecto a su uso administrativo por parte del Gobierno departamental, las provincias tienen antecedente en la Ordenanza 037 de 1991, que estableció la asociación de los 123 municipios de Boyacá, y determinó unas pautas organizacionales para propiciar un adecuado funcionamiento. Además, desde 1995 y por Decreto 1509 del 27 de diciembre, algunos municipios que pertenecían a la provincia de Sugamuxi y a la provincia de Valderrama se integraron a la provincia de La Libertad. Así mismo, se han creado otras provincias que han tenido un uso intermitente, estas son: la provincia de Puerto Boyacá y la provincia de Cubará (Ocampo, 1997, p. 89).

\section{La provincia como contenido: una herencia de la construcción histórica departamental}

Las provincias en Boyacá también son el reflejo del uso que a lo largo de la historia le han dado los grupos humanos asentados en el territorio, tanto los moradores prehispánicos como los poseedores hispánicos participaron en la configuración de las provincias.

Una herencia de la organización territorial y político-administrativa prehispánica. Debemos entender que la instauración de un sistema de administración particular por parte del Imperio español en sus dominios de la mar océano, no fue incongruente respecto al modo como era explotado u ocupado por los nativos americanos.
Como lo indican Herrera y Bonnet (2001, p.29) según Jaime Jaramillo Uribe en su artículo Nación y región en los orígenes del Estado nacional en Colombia, las primeras divisiones adoptadas en el periodo colonial "no fueron arbitrarias", sino que "correspondían a la existencia real de ciertas diferencias de geografía, riquezas naturales y aún antecedentes de población y cultura prehispánica”. Por ello, la correlación entre el llamado Nuevo Reino de Granada y el Reino de los Chibchas, es un elemento de análisis importante para entender que las construcciones espaciales y regionales no parten de un vacío histórico, sino que provienen de territorios creados que evolucionan y cuyos límites terminan por transformarse y por nominarse de manera diferente.

Además y según revisión hecha por Alexander Uribe de la obra Historia de la subregionalización de los Santanderes:

En lo que se refiere a los Santanderes y Boyacá, las provincias existentes desde la época colonial, como Guane, del Carare, del Rincón de Vélez, de las quebradas del Tequia, de los Muzos, de los Teguas, entre otras, no respondían solamente a entidades territoriales, sino a entidades humanas distintas que ocupaban lugares determinados. Ésta "subregionalización" del universo social étnico, por medio del término provincia, habla más del conjunto social que del simple territorio, contrario a como fue entendido por muchas constituciones de la época republicana, incluyendo la de 
1991. Dicen los investigadores, apoyados en las crónicas de Fray Pedro Simón, que la provincia estaba unida al grupo étnico que poblaba el territorio. Esta concepción explica el sentido de pertenencia que se tiene por la provincia en la sociedad de varios departamentos en los que subsiste la figura. (Uribe, 2009, p. 14).

Entonces, el departamento de Boyacá, en la delimitación de su territorio, se puede rastrear en las tierras donde floreció la cultura muisca o chibcha y dentro del área de influencia del Cacicato del Zaque que junto con el Cacicato del Zipa fueron usos territoriales indígenas que aprovecharon los españoles para establecerse.

Una herencia de la organización territorial y político-administrativa hispánica. Desde el siglo XVI, el desenvolvimiento histórico de Boyacá está relacionado directamente con la coyuntura histórica mundial de la expansión europea, y el establecimiento de provincias fue uno de los mecanismos que utilizó la metrópoli para institucionalizar su dominio transoceánico.

El concepto de provincia se remonta al Imperio romano y se arraigó en el actual territorio que ocupa Colombia, a través de instituciones coloniales, como las gobernaciones de las que se derivaron las provincias que se asentaron en los centros urbanos de mayor relevancia en el territorio nacional. Entre 1717 y 1810 el Virreinato de la Nueva Granada contó con 24 provincias subdivididas en cantones y parroquias. Posteriormente, el territorio del Virreinato adquiere el nombre de Confederación de Provincias Unidas de la Nueva Granada (1810-1819) constituida por 15 provincias (Rodríguez, 2007); como se ve, se conserva el uso de las provincias luego de la independencia aunque esta fue acompañada y en ciertas épocas reemplazada del todo por otras formas de organización también propias de la forma de organización territorial europea.

En 1821 en el Congreso de Cúcuta surgió el departamento de Boyacá, los departamentos estuvieron acompañados de figuras como las provincias, los cantones y las parroquias, y el departamento de Boyacá estaba integrado por las provincias de Tunja, Pamplona, Socorro y Casanare. A partir de 1824 aparecen los departamentos (doce en total) que se subdividen a su vez en provincias, cantones y parroquias; la provincia mantuvo un notorio protagonismo hasta el establecimiento de la República de la Nueva Granada (1830-1856). En ese momento, se suprimen los departamentos existentes y se fortalecen las provincias que junto a los distritos parroquiales, anteriormente parroquias, constituyeron la estructura básica de la organización territorial de la época. (Rodríguez, 2007)

Más tarde, entre 1857 y 1863, opera la Confederación Granadina que restablece los estados soberanos, integrados por provincias y distritos parroquiales, que son finalmente suprimidos en 1886. 
La provincia en Boyacá: unidad territorial, histórico-funcional de planificación en la gestión ... Lorena Andrea Estupiñan Pedraza

En 1857, el Estado soberano de Boyacá estaba conformado por las provincias de Tunja, Tundama y Casanare y los cantones de Chiquinquirá y Vélez. De los cuatro departamentos existentes en 1857 se pasó a seis en 1863: Casanare, Tundama, Norte, Occidente, Oriente y Centro.

La Constitución de 1886, de carácter centralista, dividió el país en departamentos, estos en provincias y estas en municipios. Las provincias fueron suprimidas en 1911 por Decreto Ejecutivo 306 y varios municipios le fueron segregados a Boyacá para constituir la comisaría de Arauca. Posteriormente, los municipios pertenecientes a la Orinoquía "formaron la intendencia de Casanare siendo su territorio anexado nuevamente a Boyacá hasta el año de 1973, cuando de nuevo se le segregaron otros territorios" (Gobernación de Boyacá, 2008).

De lo dicho se infiere, que la división del territorio boyacense en provincias, es el reflejo de "las formas de adaptación y transformación del medio por parte de sus gentes, descendientes de chibchas y españoles" (Ocampo, 1997, p. 59-60) y que le otorgan una identidad propia que lo diferencia de otras regiones de Colombia; dicha identidad lleva implícita la fuerza de la integración que impulsa procesos de diversidad en la unidad, de la visión de las partes que no se conciben sin su conjunto y del conjunto que no se concibe sin las partes.

\section{LA PLANIFICACIÓN EN LA GESTIÓN DEL DESARROLLO REGIONAL ENDÓGENO}

En este apartado se exponen los elementos conceptuales y teóricos que permiten entender el rol de la provincia para la planificación en la gestión del desarrollo regional endógeno. Para esto, se expone el concepto de planificación y después su lugar en el proceso de gestión. Asimismo, se presenta el enfoque del desarrollo regional endógeno, y se diferencia entre el concepto de desarrollo regional y el enfoque teórico. Estos elementos facilitarán el análisis que se desarrolla en el apartado final.

\section{La planificación}

La planificación es un método de intervención para producir un cambio en el curso de los eventos. Por ello, cuando se aplica a la conducción de la sociedad, está influida por las ideas que la orientan y está limitada por las condiciones naturales en que se desarrolla. La planificación debe llevar al buen uso del territorio geográfico y social.

La planificación ayuda a formular con coordinación los planes, los programas y los proyectos de las regiones. Además y como se indica en Ensayos sobre planificación regional del desarrollo (ILPES, 1982) la planificación del desarrollo regional destaca más la interdependencia de las localidades de una región y es posible coordinar mejor 
su desarrollo. Por ello es importantísimo organizar el desarrollo regional futuro siguiendo determinadas directrices que han sido planificadas desde los territorios.

La planificación y la participación. La planificación debe partir de la generación de consensos que permitan la organización de recursos y producción de resultados de impacto en el desarrollo individual y colectivo. Por lo anterior, una de las características del proceso de planificación es la Participación Efectiva. El proceso de Planificación debe brindar la posibilidad real de intervención a todos aquellos que representan grupos de interés, debe considerar las opiniones e intereses de los diferentes actores con el fin de priorizar problemas y acciones y lograr el máximo nivel de beneficio social posible y debe permitir llegar a acuerdos y definir propósitos, objetivos y metas compartidas, de acuerdo con la visión de futuro concertada (Departamento Nacional de Planeación, 2008).

\section{La planificación y los planes de desarrollo.} Como ya se indicó, es importante organizar el desarrollo regional futuro. Si dicha organización toma la forma de metas cuantitativas, que deberán alcanzarse en un lapso previsto y describen los medios para lograr esas metas, el conjunto de directrices suele llamarse plan de desarrollo. Los planes de desarrollo tienen una importancia significativa, ya que según sus disposiciones y métodos de preparación y ejecución "pueden servir como medios para unificar el país bajo una dirección central sin destruir la identidad de las regiones o para conseguir la descentralización del poder y la autoridad sin poner en peligro la unidad nacional" (ILPES, 1982, p.33); por ende, deberán tener muy en cuenta la dependencia recíproca entre las diferentes regiones y entre estas y el resto del país.

La característica más sobresaliente de los planes es "la coordinación en el espacio de las distintas actividades económicas y sociales del futuro" (ILPES, 1982, p. 30) Por ende, se convierte en un instrumento eficaz para señalar a las autoridades nacionales de una manera consistente, las necesidades y las posibilidades del desarrollo local. Son también características de los planes de desarrollo: la pertinencia, la articulación, la estructuración, la integralidad, la viabilidad, la concertación y la posibilidad de ser evaluados (Departamento Nacional de Planeación, 2008).

\section{La planificación como estadio de la gestión}

La planificación se integra a un proceso más extenso y profundo que es la gestión; de esta manera, la tarea de pensar el futuro se convierte en un ciclo en continuo perfeccionamiento.

La gestión debe buscar de manera eficaz y eficiente resultados frente a la reducción de la pobreza y el mejoramiento de la calidad de vida de sus habitantes $\mathrm{y}$, en consecuencia, avanzar hacia un desarrollo integral sostenible. Es un proceso sistémico, que debe contar con 
la participación de la administración, la organización política y la comunidad (Escuela Superior de Administración Pública, 2005). El buen gobierno es en definitiva el fundamento de la gestión.

La gestión incluye la planificación, pero también, tiene fundamento administrativo $\mathrm{y}$ financiero.

La gestión y la administración. Según el Ministerio de Desarrollo Económico (1997), los diferentes instrumentos de gestión, posibilitan la administración del territorio en su conjunto con fundamento en el avance de la desconcentración y la descentralización así como la coordinación de la oferta pública de bienes y servicios. Desconcentración significa situar recursos, responsabilidad por funciones y capacidad ejecutora en dependencias o lugares geográficos alejados del nivel central, aunque manteniendo una subordinación jerárquica de tales unidades a la autoridad central que encomienda esas labores. La descentralización territorial implica avanzar en un modelo de gestión que da un mayor protagonismo en las decisiones a los niveles subnacionales y a la población. La transferencia de capacidad de toma de decisión y de ejecución a otros niveles se considera necesaria para resolver los problemas en el territorio en el que se producen (Gobierno de Chile, 2009).

Del mismo modo, es fundamento administrativo de la gestión el coordinar la oferta pública de bienes, servicios y productos en el territorio, a fin de garantizar que estos sean oportunos, complementarios, de calidad y se provean en la secuencia adecuada en el caso que se trate de bienes que se encuentren en una cadena de insumo-producto.

La gestión y la financiación. La gestión incluye la financiación, que comprende el manejo de aquellos recursos que son generados y ejecutados en función del desarrollo. La gestión financiera se define: como el manejo financiero desde el punto de vista de la planificación de recursos, la ejecución, seguimiento y evaluación y finalmente la forma de consecución de otros recursos. La determinación o definición financiera del territorio es condición sine qua non para ejecutar el plan (Ministerio de Desarrollo Económico, 1997).

Así, se obtiene una mayor eficiencia de los recursos públicos al sincronizar y complementar las acciones en el territorio. Del mismo modo se logra dar mayor eficacia a la ejecución del presupuesto público, pues las acciones de servicios complementarios se apoyan y potencian y no se obstaculizan. La gestión debe orientarse a la formulación de presupuestos regionales que sean la expresión financiera de los planes y programas de los servicios que tienen presencia regional o proveen bienes y servicios en el territorio, ajustados a la política nacional de desarrollo y al presupuesto de la nación (Gobierno de Chile, 2009).

Así, es definitivo planificar y gestionar de manera paralela, como indica Pogiese 
(1993), estos procesos son claves para el cambio de las condiciones de vida que se intentan transformar.

\section{El desarrollo regional endógeno}

En este documento se hace énfasis en la organización de los territorios ${ }^{4}$ como uno de los factores que determina el desarrollo regional, teniendo en cuenta que "El territorio organizado constituye la base doméstica donde se construye la ventaja competitiva" (Baena, Sánchez \& Montoya, 2006, p. 177-178). Este énfasis es comprensible desde el enfoque del desarrollo regional endógeno.

Sin embargo y antes de definir en qué consiste este enfoque, es necesario entender qué es el desarrollo regional.

El desarrollo regional. Es un proceso de cambio social sostenido que apunta al perfeccionamiento de la región, de la comunidad de personas que constituyen la sociedad regional y de cada individuo miembro y del territorio que ocupan. Como lo afirma Hilhorst (citado por ILPES, 1982, p. 53), un aumento en el bienestar de la región debe verse expresado en "indicadores como el ingreso por habitante, disponibilidad de servicios, adecuación del sistema legal-administrativo, etc. La visión del desarrollo regional permite ocuparse de las regiones diferencialmente y por consiguiente de sus problemas particulares sin perder la perspectiva nacional".

Las regiones juegan un papel creativo, como indica Stöhr (citado por ILPES, 1982), al presentarse como instrumentos de la toma de decisiones para la participación activa de las poblaciones en la definición y ejecución de una combinación de políticas, planes y proyectos de desarrollo local, regional y nacional (organizar y racionalizar mejor la presión política local para que participe en el proceso de la toma de decisión). Esta idea se basa en el supuesto de que el regionalismo -entendido como conciencia regional, patrones culturales regionales y solidaridad regional- constituye un factor positivo para el desarrollo.

Las regiones pueden justificarse en un amplio campo técnico, principalmente para la toma de decisiones y para la coordinación en el interior de la nación. Pero, además de lo técnico, la introducción del concepto regional significa, como también afirma Walter Stöhr, "formalizar y ordenar un acto mental que ya existe, retomar los regionalismos implícitos que tiene" (citado por ILPES, 1982, p. 159).

Enfoque de desarrollo regional endógeno. De manera específica, la teoría del

\footnotetext{
Organizar se refiere a disponer algo ordenadamente con miras a una función o usos determinados. Para nuestro texto, la organización del territorio se refiere al modo como la provincia, como lugar concreto al que la historia ha dado reconocimiento e importancia, se usa para la planificación tanto como escenario para la participación como para el diseño y ejecución de proyectos, según se verá más adelante.
} 
desarrollo regional endógeno le apuesta a activar las capacidades internas de las organizaciones territoriales, a movilizar el talento humano, la organización y a aprovechar la cultura y la identidad local. De esta manera, se logra el óptimo empleo de los impulsos del crecimiento así como el diseño de estrategias efectivas para disminuir los desequilibrios. El capital social, el capital humano, el conocimiento, las instituciones, están determinados dentro de la región y no de forma exógena.

El desarrollo regional endógeno incluye cuatro planos: el plano político, se refiere a la capacidad territorial para tomar decisiones relevantes, a la capacidad de diseñar y ejecutar políticas de desarrollo y a la capacidad de negociar; el plano económico, se refiere a la apropiación y reinversión in situ de parte del excedente, a fin de diversificar la economía, dándole una base de sostenibilidad al territorio en el tiempo; el plano tecnológicocientifico y el plano cultural, como matriz generadora de identidad socio-territorial.

Como se puede ver, la endogeneidad del desarrollo adquiere múltiples formas incluyendo el modo como se organiza, se identifica y se usa el territorio, porque como afirma Vázquez Barquero (citado por DNP, 2008, p. 15) la endogeneidad estaría definida por la especificidad territorial de los problemas. Y, por supuesto, la búsqueda de soluciones pasa por la potenciación de esas condiciones naturales que le son propias al territorio.
En ese sentido, un desarrollo regional endógeno debe hacer uso de la planificación.

En primer lugar, la delimitación y circunscripción de la planificación a un territorio particular y específico facilita el "qué hacer" gubernamental, al poner de precedente las necesidades y potencialidades particulares de dicha unidad territorial. El desarrollo endógeno indica que para activar las capacidades internas de las organizaciones territoriales (como el departamento) es necesario aprovechar la ubicación y el ecosistema, las instituciones, el sistema de producción, la cultura y la identidad, etc. Un territorio organizado a partir de sus peculiaridades provee "un entorno favorable para la transformación de las ventajas comparativas en ventajas competitivas sustentables" (Departamento Nacional de Planeación, 2008, p. 17).

En segundo lugar, la construcción de instrumentos, de planes de desarrollo desde una perspectiva territorial, resulta significativa para la gestión del desarrollo porque permite la articulación permanente de los procesos de planificación, ejecución y evaluación teniendo siempre presente el aprovechamiento de los recursos con que se cuenta; de este modo se facilita la identificación de "las capacidades que se encuentran ocultas, diseminadas o mal utilizadas" (Boisier, 1996, p. 6) como parte de una visión endógena.

Así, se adquiere lo que Boisier (1996) denomina (dentro del plano político 
del desarrollo endógeno) capacidad territorial para tomar decisiones, lo que significa poseer capacidad para elegir, diseñar y ejecutar (planificación-gestión) una política o políticas coherentes de desarrollo.

En principio, a lo ya expuesto se puede indicar que la visión endógena del desarrollo regional es un adecuado marco que nos permite explicar los alcances que la provincia en Boyacá tiene como unidad territorial, histórico-funcional en la planificación de la gestión del desarrollo regional endógeno.

\section{LA PROVINCIA: UNIDAD DE PLANIFICACIÓN EN LA GESTIÓN DEL DESARROLLO REGIONAL ENDÓGENO A NIVEL DEPARTAMENTAL, 2004-2011}

En este apartado se analiza el rol de la provincia como unidad de planificación en la gestión del desarrollo en los gobiernos de Jorge Londoño y José Rozo.
Antecedentes de la provincia como unidad de planificación

Los planes de desarrollo, obligatorios a partir de 1994, año en que se firma la Ley 152 Orgánica del Plan de Desarro1lo, dan cuenta de que la inclusión de la provincia como unidad territorial en los planes de desarrollo más recientes es el reconocimiento de su natural uso político y administrativo, como se puede ver en la Tabla 1.

Con excepción del plan propuesto en el trienio 2001-2003 y que carece de dimensión territorial, en Boyacá se planifica desde las provincias. Además y dependiendo de las necesidades de cada momento así como de la búsqueda de mayor efectividad, el número de provincias se incrementa para servir adecuadamente en la planificación de la gestión del desarro1lo departamental. En este mismo sentido vale la pena insistir en el gran vacío que representa la ausencia de referencias a la dimensión territorial o a la organización político-administrativa del departamento en el plan 2001-2003, un documento pobre y que carece de una visión entrañable y real de Boyacá y sus gentes. 
Tabla 1. Síntesis planes de desarrollo posteriores a la Ley 152 de 1994

\begin{tabular}{|c|c|c|c|}
\hline PLAN & $\begin{array}{c}\text { DIMENSIÓN } \\
\text { TERRITORIAL }\end{array}$ & $\begin{array}{c}\text { UNIDAD } \\
\text { TERRITORIAL }\end{array}$ & $\begin{array}{l}\text { UNIDAD HISTÓRICO- } \\
\text { FUNCIONAL }\end{array}$ \\
\hline $\begin{array}{c}\text { PLAN DE } \\
\text { DESARROLLO } \\
\text { TODOS POR } \\
\text { BOYACÁ } \\
1995-1997\end{array}$ & LA PROVINCIA & $\begin{array}{l}\text { Hábitat concreto } \\
{[\ldots] \text { compuesto }} \\
\text { por provincias }\end{array}$ & $\begin{array}{l}\text { - Canal para llevar las necesidades } \\
\text { más sentidas por los municipios y } \\
\text { las provincias al nivel de programas } \\
\text { departamentales. } \\
\text {-Territorio para la coordinación } \\
\text { e integración de sectores. } \\
\text {-Facilita el ejercicio de la } \\
\text { subsidiariedad y concurrencia. } \\
\text {-Escenario y agente del desarrollo. }\end{array}$ \\
\hline $\begin{array}{c}\text { PLAN DE } \\
\text { DESARROLLO } \\
\text { BOYACÁ NUEVA } \\
\text { HISTORIA } \\
\text { 1998-2000 }\end{array}$ & LA PROVINCIA & $\begin{array}{l}\text { El departamento } \\
\text { de Boyacá se } \\
\text { divide en } 14 \\
\text { provincias }[\ldots] \\
\text { necesidad de } \\
\text { intervenciones } \\
\text { diferenciadas en } \\
\text { las provincias. }\end{array}$ & $\begin{array}{c}\text { - En la provincia es donde se ejecutan } \\
\text { políticas, programas, acciones } \\
\text { y normativas del plan. } \\
\text { - Desde la provincia se planifica y se } \\
\text { promueve el desarrollo económico } \\
\text { y social. } \\
\text {-Lugar que facilita la coordinación, } \\
\text { concurrencia, subsidiariedad y } \\
\text { complementariedad entre las } \\
\text { entidades territoriales. } \\
\text { - Unidad territorial [...] potencial } \\
\text { para el desarrollo regional. }\end{array}$ \\
\hline $\begin{array}{c}\text { PLAN DE } \\
\text { DESARROLLO } \\
2001-2003 \\
\text { EL RENACER } \\
D E B O Y A C A ́\end{array}$ & \multicolumn{3}{|c|}{$\begin{array}{l}\text { No hay referencia a la dimensión territorial del plan y no se mencionan } \\
\text { las provincias. }\end{array}$} \\
\hline
\end{tabular}

Fuente: Elaboración de la autora, adaptado de Gobernación de Boyacá (1995, 1998, 2001).

La provincia como unidad de planificación en la gestión del desarrollo regional endógeno. Gobiernos Londoño y Rozo

Jorge Eduardo Londoño y José Rozo Millán como gobernadores de Boyacá por el Partido Verde, compartieron visiones de gobierno que quedaron plasmadas en la planificación de la gestión del departamento.
Respecto a la provincia como unidad territorial, el plan Boyacá deber de todos incluye un apartado denominado Caracterización departamental, en el que se indica que el departamento está integrado por 123 municipios distribuidos en 12 provincias. Así mismo, en el plan Boyacá para seguir creciendo, el departamento "se divide para la Gestión Territorial en 123 municipios, los cuales se agrupan en 13 provincias". 
En este plan se considera nuevamente el uso de la provincia La Libertad 5 .

Ahora y en relación con la planificación y su carácter participativo, en los dos planes se incluyeron las provincias, como territorios de encuentro para la construcción de los planes. El plan de desarrollo
Boyacá deber de todos se construyó por medio de doce encuentros provinciales que "permitieron recuperar el verdadero sentido de participación y potenciar la capacidad de influir e incidir en el diseño y en la marcha del destino colectivo" (Gobernación de Boyacá, 2005), tal y como se ilustra en la Figura 3.

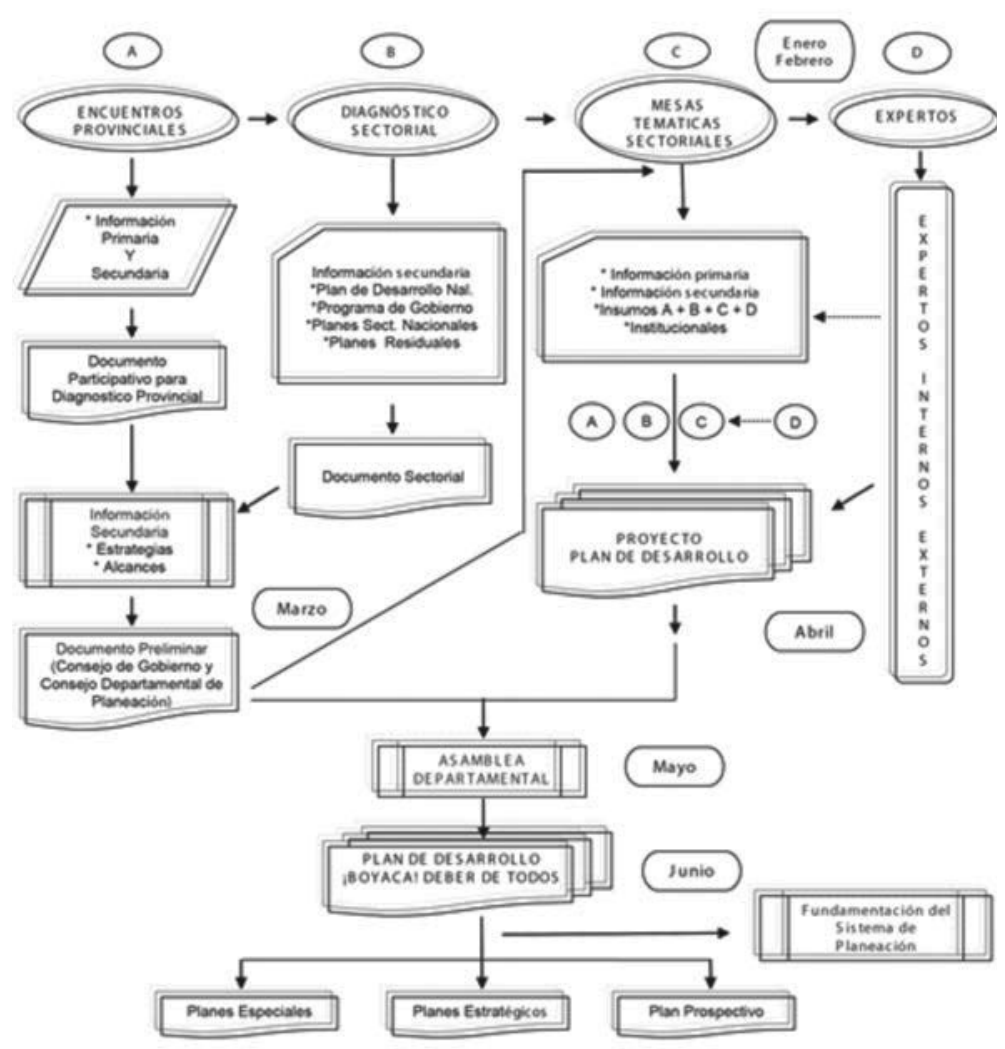

Figura 3. Proceso de formulación del plan de desarrollo.

Fuente: Gobernación de Boyacá (2005). 
Por su parte, en el plan Boyacá para seguir creciendo, la provincia fue incluida como parte del sistema de planificación territorial de Boyacá a través de los consejos provinciales, donde también se hizo un ejercicio de participación comunitaria.
Además y respecto a su rol en la gestión del desarrollo regional endógeno, las provincias tuvieron un lugar estratégico. En ambos planes fueron incluidas como parte de los denominados sistemas regionales (Figuras 4 y 5), que son proyectos para conformar un modelo regional de alto desempeño y motricidad con fuerza para integrarse a procesos con mayor avance.

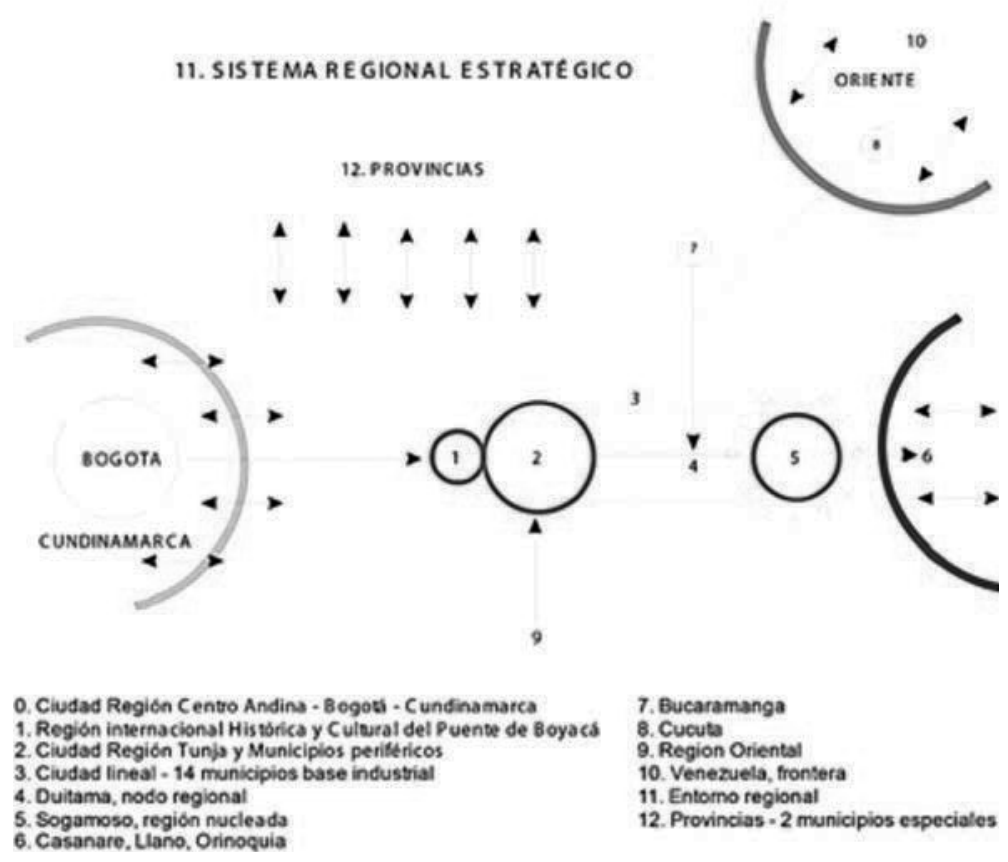

Figura 4. Sistemas regionales.

Fuente: Gobernación de Boyacá (2005). 


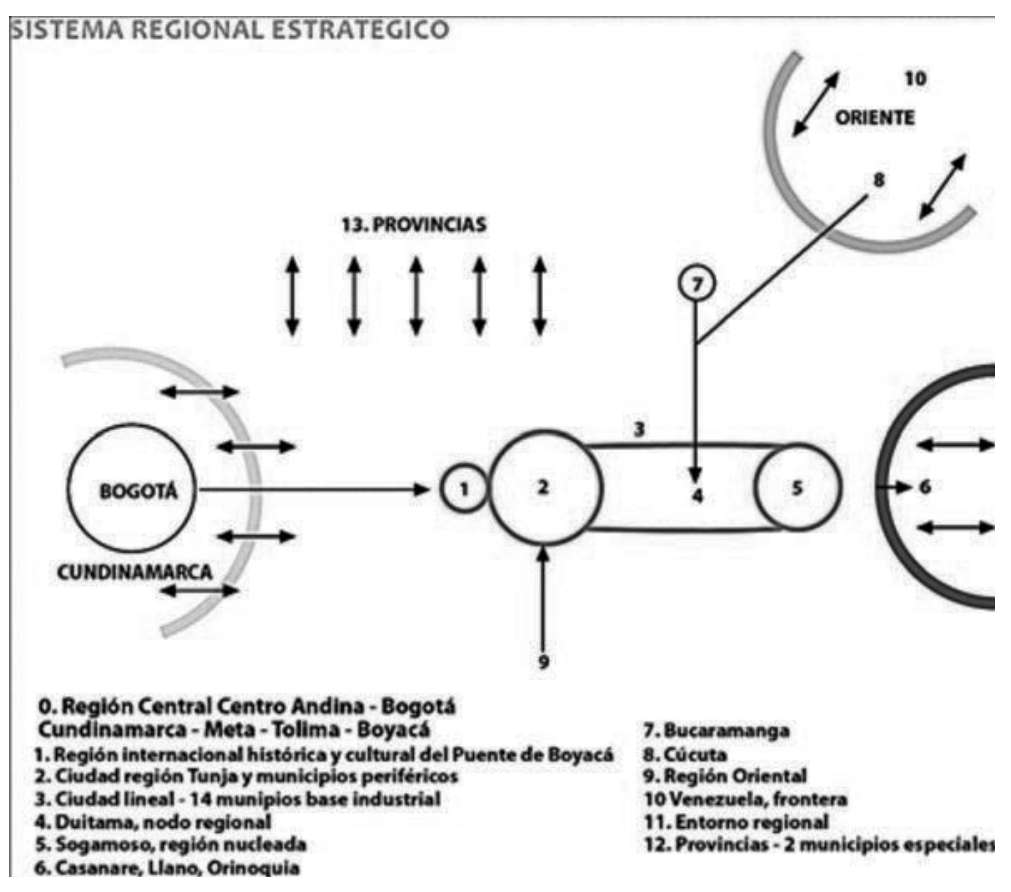

Figura 5. Sistema regional estratégico. Fuente: Gobernación de Boyacá (2008).

De manera específica y según se indica en el plan Boyacá para seguir creciendo, y tal como se puede ver en la Tabla 1 , el territorio es actor, más allá de ser soporte y contenedor de los procesos de desarrollo local y regional, integración fronteriza y competitividad internacional; por lo anterior, en este plan se incluye un aparte denominado Desarrollo provincial teniendo en cuenta que:

La provincia no es solo una unidad territorial intermedia, sino una estrategia de gestión integral para el desarrollo regional que afianza la conducta endógena a la que corresponden las jurisdicciones plenas de las Asocia- ciones de Municipalidades, buscando que tengan impacto en los proyectos de atención prioritaria del Plan. La provincia, a nuestro juicio, es un subsistema territorial, urbano regional; esto es, el conjunto de municipalidades integradas para propósito del Plan, atendiendo la historia y rasgos de homogeneidad, articulados a partir de la cabecera o capital provincial, como el principal centro administrativo y de negocios. El Desarrollo Provincial se asocia al desarrollo regional, a la búsqueda de identidades y potencialidades que permitan su diferenciación estratégica, óptimo aprovechamiento y a la articulación local de forma orgánica y 
sistémica, pero también a los proyectos susceptibles de atraer inversión propia y externa, pública y privada (Gobernación de Boyacá, 2008).

Así mismo y en relación con las funcionas otorgadas a las regiones en cuanto al Desarrollo regional (ILPES, 1982) y las funciones identificadas para las provincias en los planes de desarrollo, se puede ver en la Tabla 2.

Por otra parte, la provincia ha sido incluida como escenario de la gestión territorial de proyectos. El banco de proyectos incluye varias categorías entre otros, macro-proyectos, proyectos sectoriales, provinciales y otros especiales, esto en relación a su ejecución. Estos proyectos aunque deben estar conectados con los planes nacional, departamental y municipales se espera tengan un impacto regional (Gobernación de Boyacá, 2005).

Entre dichos programas, desarrollados en diferentes momentos y de manera particular por cada uno de los gobiernos, podemos hacer referencia a los siguientes:

- Plan Frutícola Nacional. Desarrollo de la fruticultura en Boyacá (2006). Este plan elaborado por el Ministerio de Agricultura, la Gobernación de Boyacá, el Fondo Nacional de Fomento Hortifrutícola, la Asociación Hortifrutícola de Colombia Asohofrucol y la Sociedad de Agricultores y Ganaderos del Valle del Cauca, fue diseñado y ejecutado incluyendo también a las provincias como dimensión territorial de Boyacá:
"Pitaya amarilla: esta fruta tiene un claro mercado internacional, pero su oferta exportable es muy baja. Para Boyacá se recomiendan 500 hectáreas nuevas para sembrarlas entre 800 y 1.800 metros sobre el nivel del mar en la provincia de Lengupa, en los municipios de Miraflores, Zetaquirá, Páez y Berbeo" (PFN, 2006, p.61).

- Plan Vial Regional (2009). El diseño y ejecución del plan utiliza las provincias y las capitales provinciales como fundamentales para la definición y priorización de los ejes viales estratégicos; así mismo, al momento de contabilizar la infraestructura vial construida, esta se agrupa por provincias, como se puede ver en la Tabla 3.

- Plan Educativo "Por los senderos del saber" (2008-2011). Tal y como indica el informe de la Contraloría (2012), las instituciones educativas se agrupan provincialmente y provincialmente son intervenidas, "Se suscribió un Convenio de asociación entre departamento, asociación alianza educativa y fundación Antonio Puerto, con el objeto de fortalecer y mejorar la calidad educativa, con enfoque en derechos humanos, en niños y adolescentes de 11 Instituciones Educativas de la Provincia Gutiérrez" (p.30).

- Programa de Salud Mental (20052009). Hace un análisis de la salud mental en el departamento desde el punto de vista provincial y municipal, como se muestra en la Figura 6. 
Apuntes Cenes Vol. 33, No. 58, ISSN 0120-3053

Julio - Diciembre 2014, Págs. 163-188

Tabla 1. Síntesis planes de desarrollo posteriores a la Ley 152 de 1994

\begin{tabular}{|c|c|}
\hline \begin{tabular}{|c|} 
FUNCIONES DE LAS \\
REGIONES PARA EL \\
DESARROLLO NACIONAL \\
Y SUBNACIONAL
\end{tabular} & PLANES DE DESARROLLO DEPARTAMENTALES \\
\hline $\begin{array}{l}\text { Crear, analizar y proyectar } \\
\text { información subnacional }\end{array}$ & $\begin{array}{l}\text { Esta función se cumple al establecer por medio de ordenanza que el } \\
\text { departamento como hábitat concreto está compuesto por provincias que } \\
\text { son el "canal para llevar las necesidades más sentidas al nivel de programas } \\
\text { departamentales". Por supuesto, la información obtenida puede llevar a la } \\
\text { creación de otras provincias ya sea por cuestiones económicas, políticas o } \\
\text { socioculturales. Así, el número varía de ente } 12 \text { y hasta } 14 \text { provincias en los } \\
\text { planes de desarrollo de los últimos cinco gobiernos. }\end{array}$ \\
\hline $\begin{array}{l}\text { Relacionar los proyectos } \\
\text { específicos mediante una } \\
\text { subagregación regional }\end{array}$ & $\begin{array}{l}\text { La lectura analítica de los planes indicó que en las provincias: } \\
\text { - Se ejecutan políticas, programas, acciones y normativas. } \\
\text { - Se planifica y promueve el desarrollo económico y social. } \\
\text { - Se alcanza el desarrollo territorial sostenible. } \\
\text { - Se formulan los planes estratégicos provinciales. }\end{array}$ \\
\hline $\begin{array}{l}\text { Incorporar al proceso de } \\
\text { desarrollo los recursos } \\
\text { naturales, humanos } \\
\text { y financieros }\end{array}$ & $\begin{array}{l}\text { La provincia es sin duda alguna estrategia de gestión integral para el } \\
\text { desarrollo regional, ya que, como se indica en los planes, es escenario y } \\
\text { agente, contenedor y contenido, es importante en tanto se usa pero también } \\
\text { en consideración a quienes definen esos usos. }\end{array}$ \\
\hline $\begin{array}{l}\text { Organizar y racionalizar las } \\
\text { presiones políticas locales y } \\
\text { regionales para contribuir al } \\
\text { proceso de toma de decisión }\end{array}$ & $\begin{array}{l}\text { La provincia es parte fundamental de la construcción de los planes en } \\
\text { tanto es escenario de participación (concejos provinciales, encuentros } \\
\text { provinciales, etc.) }\end{array}$ \\
\hline $\begin{array}{l}\text { Contribuir a una mejor } \\
\text { distribución espacial de } \\
\text { las inversiones nacionales }\end{array}$ & $\begin{array}{l}\text { Los planes de desarrollo coinciden en otorgar a la provincia la función de: } \\
\text { - Ejecución de políticas, programas, acciones y normativas del plan. } \\
\text { - De planificación y promoción del desarrollo económico y social. } \\
\text {-Integrar y configurar el (los) sistema (s) regional(es) de desarrollo. } \\
\text {-Gestionar territorialmente los proyectos. }\end{array}$ \\
\hline $\begin{array}{l}\text { Coordinar la inversión } \\
\text { sectorial por regiones }\end{array}$ & $\begin{array}{l}\text { En este sentido, los planes identifican las provincias como: } \\
\text {-Territorios para la coordinación e integración de sectores. } \\
\text {-Integrante del (los) sistema (s) regional(es) de desarrollo. } \\
\text {-Figura del sistema regional estratégico del departamento. }\end{array}$ \\
\hline $\begin{array}{c}\text { Introducir criterios nacionales } \\
\text { en los esfuerzos por obtener } \\
\text { una medida de la autonomía } \\
\text { regional }\end{array}$ & $\begin{array}{l}\text { Se indica en los planes que las provincias: } \\
\text {-Facilitan el ejercicio de la subsidiariedad, coordinación, concurrencia y } \\
\text { complementariedad respecto a las entidades territoriales. } \\
\text {-Son parte del sistema de planificación territorial de Boyacá a través de los } \\
\text { encuentros provinciales y en la conformación de los consejos provinciales. }\end{array}$ \\
\hline $\begin{array}{l}\text { Combinar flexibilidad de las } \\
\text { unidades necesarias para el } \\
\text { proceso del desarrollo, con la } \\
\text { estabilidad requerida por el } \\
\text { regionalismo sociocultural y } \\
\text { la rigidez de un sistema } \\
\text { de información }\end{array}$ & $\begin{array}{l}\text { La inclusión de las provincias históricas en la planificación de la gestión } \\
\text { del desarrollo regional endógeno en Boyacá, se da por la necesidad de } \\
\text { intervenciones diferenciadas que resuelvan problemas a través del uso } \\
\text { efectivo de los recursos existentes y que se logra cuando los encargados } \\
\text { de tomar las decisiones conocen de antemano los elementos particulares } \\
\text { del escenario de su acción y por ende tienen acceso a información veraz } \\
\text { y apropiada. }\end{array}$ \\
\hline
\end{tabular}

Fuente: elaboración de la autora. 
La provincia en Boyacá: unidad territorial, histórico-funcional de planificación en la gestión ... Lorena Andrea Estupiñan Pedraza

Tabla 3. Proyectos de infraestructura vial 2011

\begin{tabular}{|l|c|c|c|c|}
\hline PROVINCIA & $\begin{array}{c}\text { Pavimentación } \\
\text { y Rehabilitación }\end{array}$ & $\begin{array}{c}\text { Mantenimiento } \\
\text { Periódico (Km) }\end{array}$ & $\begin{array}{c}\text { Mantenimiento } \\
\text { Rutinario (Km) }\end{array}$ & $\begin{array}{c}\text { Puentes } \\
\text { Construidos }\end{array}$ \\
\hline CENTRO & 5.5 & 44.95 & 464.7 & 7 \\
RICAURTE & 3.2 & 5 & 418 & 0 \\
OCCIDENTE & 2.9 & 0 & 1.012 .5 & 0 \\
NORTE & 1.74 & 0 & 248 & 0 \\
VALDERRAMA & 0 & 0 & 206 & 0 \\
LA LIBERTAD & 0 & 10 & 87 & 0 \\
NEIRA & 0 & 0 & 741.8 & 0 \\
LENGUPÁ & 0.55 & 0 & 50 & 0 \\
GUTIÉRREZ & 0.66 & 0 & 1.204 & 0 \\
SUGAMUXI & 27.4 & 0 & 428 & 1 \\
TUNDAMA & 9.63 & 0 & 245 & 2 \\
ORIENTE & 0.42 & 40 & 235 & 0 \\
MÁRQUEZ & 0.22 & 31.1 & 119 & 0 \\
TOTAL EJECUTADO & 52.220 & 131.05 & 5.459 .00 & 10.00 \\
\hline
\end{tabular}

Fuente: Contraloría General de Boyacá (2012).

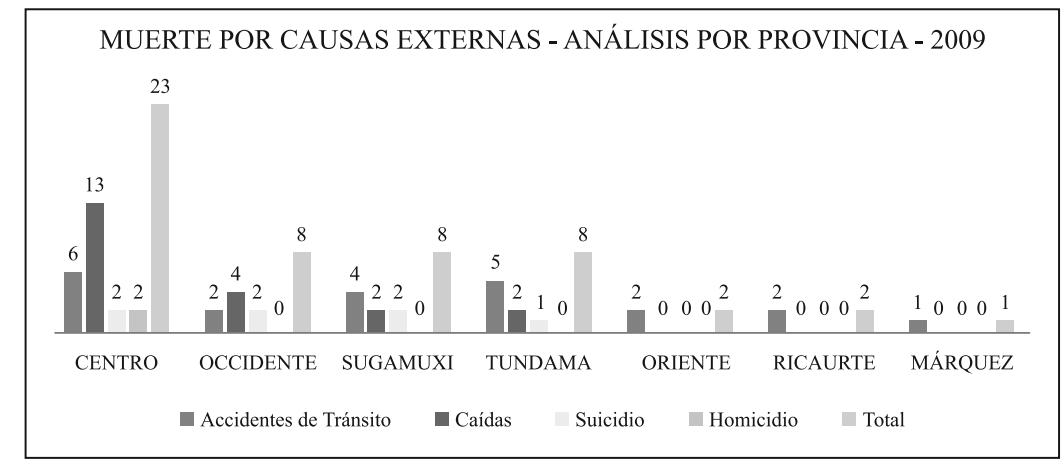

Figura 6. Muerte por causas externas, análisis por provincias 2009.

Fuente: elaborado por el equipo de trabajo de salud mental del departamento de Boyacá y adaptado por Contraloría General de Boyacá (2012).

- Unidades Desconcentradas Provinciales. (2009). Anteriormente conocidas como Direcciones de Núcleo, durante el gobierno Rozo fueron transformadas con el fin de optimizar su labor como enlace entre la Secretaría Departamental y las Instituciones Educati- vas. En este mismo sentido, se dividió el departamento en 15 provincias: Ricaurte se divide en Ricaurte Alto y Ricaurte Bajo, Sugamuxi se une a La Libertad, y se reactivan Cubará y Puerto Boyacá (Secretaría de Educación de Boyacá, 2009, p.1-5). 
- Estrategia Tierra viva (2008-2011). Desarrollada por el gobierno departamental para hacer frente a la pobreza extrema, incluye "ubicación geográfica de los más pobres, caracterización de los recursos naturales del territorio, apropiación a nivel local de la estrategia, coordinación interinstitucional para favorecer la reducción de la pobreza", desde una perspectiva provincial y municipal, según lo descrito en informes del Departamento Administrativo de Planeación, citados por Emily Belland en el documento de trabajo Dinámicas regionales, economía y pobreza: departamento de Boyacá (2013, p.28)

Así y a través de las provincias, se cumple primero con el carácter participativo y de concertación de la planificación; segundo, se facilita la gestión del desarrollo y con ella la coordinación de actividades sociales, económicas y políticas, y tercero, se refuerza la labor de los gobiernos municipales y del gobierno departamental al hacer uso de los principios de coordinación, subsidiariedad y concurrencia que existen entre los diferentes niveles de gobierno y que están consignados en la Constitución Política.

\section{CONCLUSIONES}

Para que la planificación como dimensión de la gestión conduzca al mejoramiento de las condiciones de vida de una comunidad, debe partir del uso adecuado del territorio. Y los planes como resultado del proceso de planificación, necesariamente tienen que establecerse en una dimensión territorial específica para que la perspectiva de desarrollo sea una sola. Dicha dimensión es más adecuada cuando ya existe y es mejor aún si se utiliza a largo plazo.

Como se pudo ver en cada uno de los planes analizados pero en particular en los planes recientes, la provincia es desde lo territorial, la unidad para pensar el qué hacer y el cómo hacer del gobernante boyacense. Y lo es, porque es parte de la visión comunal y compartida que tienen los ciudadanos del departamento, pero también porque administrativa y políticamente existe. Por esto resulta mucho más práctico pensar provincialmente en Boyacá.

A través de las provincias, se profundiza la identidad de la población boyacense, las características naturales de su relieve, se coordinan actividades de los municipios asociados y se impulsan proyectos departamentales, como quedó claro en cuatro de los cinco planes de desarrollo analizados. De manera particular en los planes, programas y proyectos ejecutados en los periodos 2004-2007 y 2008-2011, la provincia se utilizó para crear, analizar y proyectar información subnacional, lo que efectivamente permitió "llevar las necesidades más sentidas al nivel de programas departamentales", e incluyó la variación en el número de provincias, que según necesidad y gobierno, han sido hasta 15.

Así mismo, el aprovechamiento de la provincia como unidad territorial a la que 
la historia y su uso dan sentido, permitió incorporar al proceso de desarrollo los recursos naturales, humanos y financieros con que realmente cuenta el departamento, lo que indica que la provincia es estrategia de gestión integral para el desarrollo regional. De esta manera se logró contribuir a una mejor distribución espacial de las inversiones nacionales. Al mismo tiempo, las provincias permitieron coordinar la inversión sectorial por regiones y relacionar proyectos especificos con territorios específicos donde se ejecutaron políticas, programas, acciones y normativas particulares en salud, educación, agricultura e infraestructura vial, entre otras.

Fueron también una herramienta política, porque permitieron organizar y racionalizar las presiones políticas locales y regionales para contribuir al proceso nacional de la toma de decisión a través de los encuentros como de los diálogos provinciales, que resultaron definitivos en la construcción participativa de los planes.
Y finalmente, al combinar la flexibilidad de las unidades necesarias para el proceso del desarrollo, con la estabilidad requerida por el regionalismo sociocultural y la rigidez de un sistema continuo de información, la inclusión de las 12 provincias históricas pero también de las otras provincias (como en el caso de la educación), facilitó el desarrollo de intervenciones diferenciadas que reflejaron la racionalización de la toma de decisiones con base en información veraz y apropiada; se trata de ser realmente conscientes acerca de la manera como se ve la sociedad en Boyacá, de utilizar lo que hay del mejor modo y advertir que lo que seremos está en los que somos.

La identificación de la provincia como unidad territorial histórico-funcional de la planificación en la gestión del desarrollo regional endógeno en Boyacá, ilustra de manera contundente la correlación natural entre la organización del territorio y el desarrollo de una comunidad humana.

\section{REFERENCIAS}

Baena, E., Sánchez, J. J. \& Montoya, O. (2006). Algunos factores indispensables para el logro del desarrollo regional. Scientia et Technica, 31, 177-178.

Belland, E. (2013). Dinámicas regionales, economía y pobreza: departamento de Boyacá [en línea]. Documentos de trabajo de Rimisp. Recuperado de http://www.rimisp.org/wp-content/ files_mf/1379097015Doc21AguayPobrezaBoyaca.pdf.

Boisier, S. (1996). El vuelo de la cometa. Una metáfora para una teoría del desarrollo territorial. Recuperado de: https://www.u-cursos.cl/fau/2011/2/GEO-704/1/material.../13712.

Contraloría General de Boyacá. (2012). Situación de las finanzas públicas del departamento de Boyacá. Tunja: Contraloría General de Boyacá. 
Contraloría General de Boyacá. (2012). Seguimiento al objetivo del milenio "Mejoramiento de la salud sexual y reproductiva en Boyacá”. Tunja: Contraloría General de Boyacá.

Departamento Nacional de Planeación. (2008). Características del desarrollo territorial departamental. Recuperado de http://www.incoder.gov.co/documentos/Estrategia\%20 de\%20Desarrollo\%20Rural/Pertiles\%20Territoriales/ADR_GARCIA\%20ROVIRA/ Documentos\%20de\%20Apoyo/DNP\%20Caracterizacion\%20de\%20desarrollo\%20 territorial\%20departamental.pdf

Escuela Superior de Administración Pública-ESAP-. (1998). La planeación del desarrollo territorial. Nuevas perspectivas. Bogotá: ESAP.

Escuela Superior de Administración Pública -ESAP-. (2005). Gestión pública local. Recuperado de http://www.dnp.gov.co/archivos/documentos/DDTS_Gestion_Publica_ Territorial/ $1{ }^{\mathrm{a}}$ Gestion.pdf

Gobernación de Boyacá. (1995). Plan de Desarrollo 1995-1997 Todos por Boyacá. Tunja: Gobernación de Boyacá.

Gobernación de Boyacá. (1998). Boyacá 2000. Nueva historia. Plan de desarrollo 1998.2000. Tunja: Gobernación de Boyacá.

Gobernación de Boyacá. (2001). Plan de Desarrollo 2001-2003. El renacer de Boyacá. Tunja: Gobernación de Boyacá.

Gobernación de Boyacá. (2005). Plan de desarrollo departamental Boyacá deber de todos 2004-2007. Bogotá: Ediciones Jurídicas Gustavo Ibáñez.

Gobernación de Boyacá. (2008). Plan de desarrollo "para seguir creciendo". Recuperado de http://www.boyaca.gov.co/?idcategoria=5478\#. Plan de Desarrollo.

Gobernación de Boyacá. (2008). Ordenanza número 010 de 2008. Recuperado de http://www. boyaca.gov.co/?idcategoría $=5478 \#$.

Gobierno de Chile, División de Desarrollo Regional Departamento de Fortalecimiento Regional. (2009). Guía metodológica sistema de gestión territorial integrada. Recuperado de: http:// www.bosquesandinos.info/biblioteca/DC_024.pdf.

Herrera, M. \& Bonnett, D. (2001). Ordenamiento espacial y territorial colonial en la "Región Central" neogranadina. Siglo XVIII. Las visitas de la tierra como fuente para la historia agraria del siglo XVIII. América Latina en la historia económica, 16, 11-32.

ILPES. (1982). Ensayos sobre planificación regional del desarrollo. México: Siglo Veintiuno.

Ministerio de Justicia y del Derecho de Colombia. (1998). Constitución Política de Colombia. Bogotá: Imprenta Nacional. 
Ministerio de Desarrollo Económico de Colombia. (1997). Serie procesos de aplicación. Aproximación conceptual. Recuperado de http://www.corantioquia.gov.co/docs/PGAR/ ANALISIS/pdfs/5.\%20DIMENSION\%20URBANO_REGIONAL.pdf. Serie.

Ocampo, J. (1997). Identidad de Boyacá. Tunja: Jotamar.

PFN. (2006). Desarrollo de la fruticultura en Boyacá. Recuperado de: http://www.asohofrucol. com.co/archivos/biblioteca/biblioteca_100_BOYACA.pdf

Poggiese, H. A. (1993). Metodología FLACSO de planificación-gestión (planificación participativa y gestión asociada). Recuperado de http://www.flacso.org.ar/uploaded_files/ Publicaciones/Metodologia.FLACSO.de.planificaciongestion.pdf. Cartilla.

Rodríguez, E. (2007). Las disparidades interprovinciales en Boyacá. Revista Apuntes del CENES, 27, 205-256.

Secretaría de Educación de Boyacá. (2009). Conformación equipos provinciales. Recuperado de: http://www.sedboyaca.gov.co/descargas2011/EQUIPOS_PROVINCIALES.pdf

Secretaría de Planeación. (1998). Boyacá 2000. Nueva historia. Plan de desarrollo 1998.2000. Boyacá potencia productiva, justa y solidaria. Tunja: Gobernación de Boyacá.

Secretaría de Planeación. (1998). Boyacá potencia productiva, justa y solidaria. Tunja: Gobernación de Boyacá.

Universidad Colegio Mayor de Nuestra Señora del Rosario. (2009). Proyecto de apoyo y acompañamiento a la ciudad de Ocaña. Inédito.

Uribe, J. A. (2009). La provincia en el modelo territorial colombiano. Estado del arte. Revista virtual Via Inveniendi et Iudicandi, 8, 1-43. 